\title{
Management of obstetric postpartum hemorrhage: a national service evaluation of current practice in the UK
}

This article was published in the following Dove Press journal:

Risk Management and Healthcare Policy

17 January 2017

Number of times this article has been viewed

\author{
Bassel H Al Wattar ${ }^{1, *}$ \\ Jennifer A Tamblyn $2, *$ \\ William Parry-Smith ${ }^{2, *}$ \\ Mathew Prior ${ }^{3, *}$ \\ Helen Van Der Nelson ${ }^{4, *}$ \\ On behalf of UK Audit and Research \\ trainee Collaborative in Obstetrics \\ and Gynaecology (UKARCOG)
}

'Women's Health Research Unit, Barts and the London School of Medicine and Dentistry, Queen Mary University London, London, UK; ${ }^{2}$ Institute of Metabolism and Systems Research (IMSR), University of Birmingham, Birmingham, UK; ${ }^{3}$ Division of Child Health, Obstetrics \& Gynaecology, School of Medicine, University of Nottingham, Nottingham, UK; ${ }^{4}$ Academic Centre for Women's Health, North Bristol NHS Trust, Bristol, UK

*UKARCOG
Correspondence: Bassel H Al Wattar Women's Health Research Unit, Blizard Institute, Barts and the London School of Medicine, Queen Mary University London, London EI 2DD, UK Email b.wattar@qmul.ac.uk
Background: Postpartum hemorrhage (PPH) continues to be one of the major causes of maternal mortality and morbidity in obstetrics. Variations in practice often lead to adverse maternity outcomes following PPH. Our objective was to assess the current practice in managing PPH in the UK.

Methods: We performed a national multicenter prospective service evaluation study over one calendar month and compared the current performance to national standards for managing PPH. We used a standardized data collection tool and collected data on patients' demographics, incidence of PPH, estimated blood loss (EBL), prophylactic and treatment measures, onset of labor, and mode of delivery.

Results: We collected data from 98 obstetric units, including 3663 cases of primary PPH. Fifty percent of cases were minor PPH (EBL 500-1000 mL, $\mathrm{n}=1900 / 3613,52.6 \%$ ) and the remaining were moderate PPH (EBL >1000 to $<2000 \mathrm{~mL}, \mathrm{n}=1424 / 3613,39.4 \%$ ) and severe PPH (EBL $>2000 \mathrm{~mL}, \mathrm{n}=289 / 3613,8 \%)$. The majority of women received active management of the third stage of labor $(3504 / 3613,97 \%)$ most commonly with Syntometrine intramuscular (1479/3613, $40.9 \%$ ). More than half required one additional uterotonic agent (2364/3613, 65.4\%) most commonly with Syntocinon intravenous infusion $(1155 / 2364,48.8 \%)$. There was a poor involvement of consultant obstetricians and anesthetists in managing PPH cases, which was more prevalent when managing major PPH $(p=0.0001)$.

Conclusion: There are still variations in managing PPH in the UK against national guidelines. More senior doctor involvement and regular service evaluation are needed to improve maternal outcomes following PPH.

Keywords: pregnancy, obstetric hemorrhage, postpartum, service evaluation, collaborative, guideline, national

\section{Introduction}

Postpartum hemorrhage (PPH) remains a major cause of maternal mortality and morbidity worldwide. ${ }^{1}$ In the UK, it is the third leading cause of maternal death ${ }^{1,2}$ and the most common cause of obstetric-related intensive care admissions. ${ }^{3}$

The incidence of primary PPH continues to rise progressively in the UK, reaching as high as $13.8 \%$ in $2012-2013 .{ }^{4}$ With its multifactorial etiology, early prediction and prompt management remain key measures to reduce the incidence of $\mathrm{PPH}{ }^{5,6}$ The latest Confidential Enquiries into Maternal Deaths and Morbidity in the UK highlighted large variations in managing obstetric hemorrhage emergencies leading to increased morbidity. ${ }^{1}$ National guidelines have been developed to standardize care and provide a clear evidence-based framework for clinicians and health care workers addressing 
this important health issue. ${ }^{7}$ Nevertheless, the translation of these guidelines into clinical practice is still suboptimal.

We conducted a national service evaluation study to identify variations in practice and areas for improvement in preventing and managing primary PPH against current national standards.

\section{Methods}

We performed a national multicenter prospective service evaluation study over one calendar month (SeptemberOctober 2014). We conducted the study against a prospectively registered protocol at each local R\&D department. Our study is exempt from ethical approval by the NHS Research Ethics Committee (REC) as the data collected were part of current practice nationally.

\section{Data collection}

Data were collected by 197 specialty trainees (ST1-7) in obstetrics and gynecology across the UK. Trainees were recruited by the UK Audit and Research trainee Collaborative in Obstetrics and Gynaecology (UKARCOG) network via email invitations. The UKARCOG (a nonprofit trainee-led collaborative for research in obstetrics and gynecology in the UK) oversaw the overall conduct of the study. We coordinated the data collection and extraction through a network of 13 different regions. Each regional lead recruited trainees from their local obstetric units, coordinated data collection, and coded merged data before the final analysis phase. We merged and analyzed the data from the different regions anonymously without any patients' identifiers. We collected data using a standardized paper-based data collection tool, which was first piloted among the UKARCOG members to test its content validity and feasibility. Primary PPH cases that occurred in midwifery-led units or following a home delivery were only recorded if transferred to an obstetric unit for further management.

\section{Outcomes}

The primary outcome was the incidence of primary PPH defined as $\geq 500 \mathrm{~mL}$ after vaginal birth or $\geq 1000 \mathrm{~mL}$ after cesarean within 24 hours from delivery. We categorized PPH cases per volume of estimated blood loss (EBL) to minor $(500-1000 \mathrm{~mL})$ and major $(>1000 \mathrm{~mL})$, with the later subdivided to moderate (1000-2000) and severe (>2000 mL) as per the UK Audit and Royal College of Obstetricians and Gynaecologists (RCOG) guideline.?

We collected the following secondary outcomes: patients' demographics, predefined risk factors for $\mathrm{PPH}$, onset of labor, mode of delivery, place of birth, PPH management methods and prophylactic measures, uterotonic agents used and their administration order, intravenous (IV) fluids, and blood products use and birth weight. All outcomes were defined as per the RCOG Green-top Guidance for the prevention and management of PPH. ${ }^{7}$

\section{Data analysis}

We used an imputation analysis pattern to investigate the loss of data. Cases with more than $50 \%$ loss of data were excluded. We used multiparameter imputations to compensate for missing data (up to $>13 \%$ ) for continuous outcomes. We reported the mean and standard deviation for parametric data and median and standard error for nonparametric data. We used the chi-square test to determine the distribution significance for categorical data. We used a multinomial logistic regression analysis to investigate factors affecting the severity of PPH. All statistical analyses were performed using Statistical Package for the Social Sciences (SPSS) statistical software (v19-IBM Corp.) and Microsoft Excel (2007 - Microsoft).

\section{Results}

Data were collected from 98 obstetric units including 3663 cases of primary PPH. We excluded 50 cases due to more than $50 \%$ loss of data. Table 1 illustrates the total number of women recruited from each region in the UK.

\section{Maternal demographics}

The median maternal age was 30 years (range $13-49$ years), median body mass index was $25 \mathrm{~kg} / \mathrm{m}^{2}\left(7-71 \mathrm{~kg} / \mathrm{m}^{2}\right)$, median gravidity was $2(1-12)$, and median parity was $1(0-9)$. There were 91 twin pregnancies included in the data set $(91 / 3613$, $2.5 \%$ ), and the remainder were singleton pregnancies.

Table I Number of primary postpartum hemorrhage cases recorded from the different UK regions

\begin{tabular}{ll}
\hline Regions & Number of patients \\
\hline East Midlands & 496 \\
Mersey & 218 \\
Northern & 17 \\
Northwest & 446 \\
Oxford & 411 \\
Scotland & 641 \\
Severn & 251 \\
Wales & 131 \\
Wessex & 108 \\
West Midlands & 342 \\
Yorkshire and the Humber & 444 \\
Northern Ireland & 158 \\
Total & 3663 \\
\hline
\end{tabular}


The majority of PPH cases occurred in obstetric units (3367/3613, 93.1\%). Five percent of the women delivered in a midwifery-led birth center $(186 / 3613,5.6 \%)$ and $0.7 \%$ delivered at home or before arriving to the hospital (27/3613, $0.7 \%$ ). About half of the included women had a spontaneous onset of labor (1978/3613, 54.7\%); 36.4\% were induced $(1314 / 3613,36.4 \%)$ and $7.8 \%$ underwent an elective cesarean section $(281 / 3613,7.8 \%)$. The most frequent method of induction utilized was prostaglandin agents $(982 / 1314$, $74.7 \%$ ), followed by artificial rupture of membranes (207/1314, 15.7\%). The remaining cases were induced using various other methods, such as balloon catheter.

About half of the women included achieved a normal vaginal delivery $(1662 / 3613,46 \%)$. A third had an instrumental delivery $(1102 / 3613,30.5 \%)$ and the remaining $15.7 \%$ delivered via emergency cesarean section (568/3613, $15.7 \%)$ or elective cesarean section $(281 / 3613,7.8 \%)$. A third of women received an episiotomy (1287/3613, 35.6\%) and only $5 \%$ suffered from an obstetric anal sphincter injury $(211 / 3613,5.8 \%)$.

The majority of women delivered a baby weighing between 2.1 and $3.9 \mathrm{~kg}(2938 / 3613,81.3 \%)$. In $16.7 \%$ of cases, the birth weight was $\geq 4 \mathrm{~kg}(604 / 3613,16.7 \%)$, and in a minority of cases, it was $\leq 2 \mathrm{~kg}(52 / 3613,1.4 \%)$.

\section{Prevention and management of PPH}

Fifty percent of cases were minor PPH (EBL 500-1000 mL, $\mathrm{n}=1900 / 3613,52.6 \%)$ and the remaining cases were major PPH ( $>1000 \mathrm{~mL}, \mathrm{n}=1713 / 1613,47.4 \%)$. These included moderate PPH (EBL $>1000$ to $<2000 \mathrm{~mL}, \mathrm{n}=1424 / 3613$, $39.4 \%$ ) and severe $\mathrm{PPH}$ in $8 \%$ of cases (EBL $>2000 \mathrm{~mL}$, $\mathrm{n}=289 / 3613,8 \%$ ).

The majority of women were offered and received active management of the third stage of labor (3504/3613, 97\%). The most frequently utilized prophylactic uterotonic agent was Syntometrine intramuscular (IM, 1479/3613, 40.9\%), followed by Syntocinon IV infusion (977/3613, 27\%).

More than half of the included women required at least one additional pharmacological uterotonics to control the PPH $(2364 / 3613,65.4 \%)$. Of this, 59\% had a major PPH $(1415 / 2364,59.8 \%)$, which was statistically significant $(p=0.0001)$. A third required two or more agents $(1181 / 3613$, $32.75 \%$ ) with two-third of cases associated with a major PPH $(843 / 1181,71.3 \%, p=0.0001)$. The most frequently utilized first additional uterotonic agent prescribed was a Syntocinon IV infusion (1155/2364, 48.8\%). Only 100 cases required further surgical treatment for the PPH (100/3613, 2.7\%) with balloon tamponade used in two-third of cases $(69 / 100$,
$69 \%$ ). Six women underwent a hysterectomy to control their $\mathrm{PPH}(6 / 3613,0.1 \%)$, and 13 required a laparotomy with extra sutures or artery ligation $(13 / 3613,0.3 \%)$. There were no maternal mortality cases in our sample.

A fifth of all women with PPH had a second cannula insertion documented (712/3613, 19.7\%), and about twothird of women had a urinary catheter inserted (2438/3613, 67.5\%). The Modified Early Obstetric Warning Score charts were used in the majority of cases (3041/3613, 84.2\%), and only $2.4 \%$ of cases were admitted to the intensive care unit for further monitoring $(88 / 3613,2.4 \%)$.

Obstetric consultants were involved in the management of $27.2 \%$ of PPH cases $(989 / 3613,27.2 \%)$. Consultants were more likely to be involved in major PPH cases (74\% of cases, $p=0.0001$ ). Consultant anesthetists were less involved in managing PPH cases $(616 / 3613,16.9 \%)$ and were also more likely to be involved in major PPH cases $(84 \%$ of cases, $p=0.0001$ ).

\section{Factors affecting the severity of PPH}

Table 2 highlights the relative risk of developing major PPH across different risk factors. The risk was highest for women who underwent cesarean section (2.58 for emergency sections and 1.76 for elective ones), women who delivered in an obstetric unit (risk ratio $[R R]=1.27,95 \%$ confidence interval $[\mathrm{CI}]=1.08-1.49$ ), women with a body mass index of $>30 \mathrm{~kg} / \mathrm{m}^{2}(\mathrm{RR}=1.19,95 \% \mathrm{CI}=1.10-1.29)$, and those

Table 2 The relative risk of major PPH across different risk factors

\begin{tabular}{|c|c|c|c|c|}
\hline Risk factor & $\begin{array}{l}\text { Major } \\
\text { PPH }\end{array}$ & $\begin{array}{l}\text { Relative } \\
\text { risk }\end{array}$ & $\begin{array}{l}95 \% \\
\text { confidence } \\
\text { intervals }\end{array}$ & $p$-Value \\
\hline Induction of labor & 279 & 1.01 & $0.94-1.08$ & 0.78 \\
\hline Prolonged labor ( $>12$ hours) & 292 & 0.98 & $0.89-1.08$ & 0.76 \\
\hline Spontaneous vaginal delivery & 548 & 0.55 & $0.5 \mathrm{I}-0.59$ & $<0.0001$ \\
\hline Instrumental delivery & 329 & 0.54 & $0.49-0.59$ & $<0.0001$ \\
\hline Emergency cesarean section & 557 & 2.58 & $2.46-2.70$ & $<0.0001$ \\
\hline $\begin{array}{l}\text { Elective cesarean } \\
\text { section }\end{array}$ & 279 & 1.76 & I.70-I.82 & $<0.0001$ \\
\hline Birth weight $<2 \mathrm{~kg}$ & 38 & 1.55 & $1.31-1.83$ & $<0.0001$ \\
\hline Birth weight $2-4 \mathrm{~kg}$ & 1325 & 0.78 & $0.72-0.84$ & $<0.0001$ \\
\hline Birth weight >4 kg & 338 & 1.22 & $1.12-1.32$ & $<0.0001$ \\
\hline Obstetric unit & 1620 & 1.27 & $1.08-1.49$ & 0.0040 \\
\hline $\begin{array}{l}\text { Active management of third } \\
\text { stage of labor }\end{array}$ & 1662 & 1.01 & $0.82-1.24$ & 0.89 \\
\hline Episiotomy & 369 & 0.49 & $0.45-0.54$ & $<0.0001$ \\
\hline Maternal age $>40$ years & 68 & 1.17 & $0.99-1.38$ & 0.054 \\
\hline $\mathrm{BMI}>30$ & 420 & 1.19 & $1.10-1.29$ & $<0.0001$ \\
\hline Grand multiparity $(>4)$ & 21 & 1.19 & $0.90-1.59$ & 0.20 \\
\hline
\end{tabular}

Abbreviations: BMI, body mass index; PPH, postpartum hemorrhage. 
who delivered babies weighing $<2 \mathrm{~kg}(\mathrm{RR}=1.55,95 \%$ $\mathrm{CI}=1.31-1.83)$ or $>4 \mathrm{~kg}(\mathrm{RR}=1.22,95 \% \mathrm{CI}=1.12-1.32)$. Active management of the third stage of labor did not reduce the risk of major PPH (RR=1.01, 95\% CI=0.82-1.24).

\section{Discussion}

\section{Summary of findings}

Our study provides a snapshot assessment of the current practice in the prevention and management of PPH across $55 \%$ of obstetric units in the UK. ${ }^{8}$

Overall, the rate of induction of labor, modes of delivery, and obstetric anal sphincter injuries were all within expected national figures supporting the validity of our cohort. ${ }^{9}$

There was an overall good adherence to providing active management of the third stage of labor (97\%); this, however, did not seem to significantly reduce the severity of PPH in our cohort. Contrary to national guidance, the use of Syntometrine IM was preferred to Syntocinon IM. A Cochrane review has confirmed the similarity of effect for both Syntometrine and Syntocinon when used in prophylactic setting; however, Syntometrine has significantly higher side effects (nausea, vomiting, and high blood pressure). ${ }^{10}$ This demonstrates poor adherence to national guidelines and the need to change local policy.

The use of additional uterotonic agents was generally reserved for more severe PPH cases $(p=0.0001)$ with Syntocinon IV as the preferred additional method. While IV infusion is readily available in the UK, it might not always be available in low-income settings. Other agents, such as Misoprostol and Carboprost, might offer a more costeffective alternative. ${ }^{11}$ This is currently being investigated via a network meta-analysis to shed more light on the efficacy and cost-effectiveness of the various uterotonic agents. ${ }^{12}$ The use of surgical treatments was not common; this could be due to the limited number of massive PPH cases captured in our cohort.

Adherence to using Modified Early Warning System (MEWS) charts was good (84\%); however, national guidance prompts practitioners to use them when managing all PPH cases. ${ }^{7}$ Other supportive measures, such as the use of a second cannula and urinary catheter, were rather suboptimal. Adherence to these simple and effective interventions can be improved by introducing and regularly auditing standardized PPH management protocols. ${ }^{13}$

Consultant involvement in managing PPH cases was generally poor for both obstetrics and anesthetics. The RCOG guidance advocates the involvement of senior staff in managing obstetric hemorrhage as it is likely to reduce morbidity. A future step would be to audit the grade of the trainee managing the PPH cases and contrast this to the PPH severity and timing of consultant involvement.

A number of risk factors associated with higher severity of PPH were demonstrated in our cohort. These are in line with factors established in the literature. ${ }^{14}$

\section{Strengths and limitations}

To our knowledge, our study is the first to provide a UKwide review on the management of PPH led by trainees in obstetrics and gynecology. We followed a prospective protocol that was registered at every participating obstetric unit in $R \& D$ department. We used a prospective design with a standardized data collection tool coupled with robust data coding and anonymization process to reduce data loss and selection bias. We used multiple statistical imputations to compensate for the minor data loss.

Our findings suffer from a number of limitations. The lack of funding has reduced our ability to conduct further quality assurance and resolve missing data queries. A small number of cases evaluated suffered from the high loss of data and had to be excluded from the analysis. We did not collect data on patients who did not suffer from a PPH to provide a control medium. The observational design of the study has an inherent patient selection bias and could affect the factors associated with PPH severity. We aimed to collect data from all regions in the UK, but three regions were not included due to the lack of interest in the project (London; Peninsula and Kent, Surrey and Sussex).

\section{Implication for clinical practice}

Our findings reflect a high level of awareness in preventing and managing PPH in the UK with an overall good adherence to evidence-based practice. We highlight a number of key evidence-based recommendations that were not fully complied with a national level, such as better senior doctor involvement in managing PPH and the use of prophylactic Syntocinon instead of Syntometrine as a first-line prophylactic drug. These findings should be further evaluated at the local level to introduce tailored quality improvement measures. Conducting similar projects on yearly or biyearly basis would help to assess the improvement in the quality of care and the effect of implemented measures.

Our initiative could be implemented on a wider scale including more obstetric units over a longer period of time with an appropriate control comparison subject to funding availability. This is likely to lead to more meaningful results and greater impact on patient care, with the added opportu- 
nity to evaluate current trends in the surgical management of PPH in the UK.

There is a need for a validated prediction model that could highlight patients at high risk and allow for optimized PPH management. ${ }^{15}$ Staff training and education via simulationbased learning could add great benefit to improving practice. ${ }^{16}$ Identifying auditable standards is also essential to regularly assess and update local practice.

Reducing the incidence of PPH and optimizing its management remain a great challenge for obstetric practitioners. Evidence-based interventions are needed to improve care and patient outcomes.

\section{Conclusion}

There are still variations in managing PPH in the UK against national guidelines. More senior doctor involvement and regular service evaluation are needed to improve maternal outcomes following PPH.

\section{Acknowledgments}

UKARCOG research collaborators: Adam Tyler, Aisha Baldo, Alexandra Rice, Alison Torrens, Alix Bullock, Amanda Airey, Amena Shelleh, Amna Malik, Amy Stewart, Angharad Care, Anna Clare, Anne-Marie O’Neill, Anoop Rehal, Anthony Walmsley, Anuradha Shajpal, Ashleigh Haken, Baljinder Chohan, Bansari Trivedi, Ben Luke Choo, Cameron Hinton, Camilla Lyon-Dean, Carlos Carrasco, Cecilia Iyasere, Chaitra Hirae, Christina Aye, Christine Foster, Christopher Yau, Claire Dougan, Claire Park, Clare Shakespeare, Constance Raine, Cressida Bond, Dalia Sikafi, David Ankers, David Jeevan, Deborah Bower, Dhivya Chandrasekaran, Donna Fincham, Dorreh Charlesworth, Edwin Omih, Efterpi Tingi, Elinor Edwards, Elizabeth Medford, Elizabeth Norris, Ellissa Baskind, Emily bailie, Emily Frier, Emma Laura Jones, Emma Long, Emma O'Connor, Eteyen Adegoke, Eva Myriokefalitaki, Fadi Sbeihi, Fahima Ali, Faryal Nizami, Felicity Illingworth, Fiona Day, Fiona Ross, Florence Mukuna, Gemma Brierley, Gemma Ferguson, Gemma Mcleod, Gemma Ulivi, Georgiana Ene, Gordon Buchanan, Grisham Smotra, Guy Morris, Hani Shuheibar, Hari Muppala, Harriet Lamb, Harriet Percival, Hashim Bhatti, Hawra Badri, Helen Goodall, Helen Preston, Hilary Kok, Hiranmayi Muddada, Ibrahim Elhag, Jane Creighton, Jennifer Allison, Jennifer Hartley, Jennifer Reilly, Jenny Barber, Jenny Eastwood, Jill Sturt, Jo Roper, Joanna Smith, Joanne Sentence, Joy Troko, Karen Austin Smith, Karen Meadley, Kate Alldred, Kate Danby, Kathleen Merrick, Katie McBride Katy Hall,
Kayley Towse, Kennedy Price, Kirsten Allen, Kirstin Silf, Laura Woodhouse, Leena Thomas, Lesley Curry, Lisa Scott, Lorna Hutchinson, Lucy Coyne, Lydia Gallagher, Lynne Warrander, Magdalena Thiel, Maisoon Elshtwei, Maja Kotlinska, Malathy Arun, Mandira Hazra, Mari Isdale, Marie O’Sullivan, Mark Davey, Martin Maher, Matthew Stanford, Maximiliane Kellner, Meenu Sharma, Michael Graham, Michelle Cooper, Michelle Creed, Mittal Patel, Mohamed Waseem Osman, Moira Doherty, Nadia Cassim, Naila Iftikhar, Naomi Griffiths, Natalie Boyd, Natalie Grant, Natalie Woodhead, Natasha Ng, Nav Kaur, Neesha Ridley, Neil Ryan, Neveen Khan, Nicola Cawley, Nicola Robinson, Nikola Curry, Niranjani Ramachandran, Noreen Haque, Nowmi Zaman, Olivia Hatton, Oral Doyle, Patricia Seddon, Payam Davoudian, Peter Wilson, Priya Deol, Priya Seetharaman, Rachel Squires, Razia Makander, Rebecca Fletcher, Ritu Painully, Roseanna Metcalfe, Roshni George, Rosie Malhass, Ruvana Tabassum, Ryan Edgar, Sabina Nistor, Sally Wood, Samantha Jones, Sanyal Patel, Sarah Abul-Ainine, Sarah Barker, Sarah Bell, Sarah Hobern, Sarah Ingamells, Sarah Seacombe, Sarulatha Sailesh, Seena Radhakrishnan, Shaista Iftikhar, Shaun McGowan, Siobhan Wilson, Sneha Vimalamma, Somia Khalid, Stephen O’Brien, Sudeepthi Kakara, Sumana Narain, Sumeet Tuteja, Sunneva Gilmore, Susan Tracey, Suzanna Dunkerton, Suzi Addley, Tamara Abdul Raheem, Thomas Mosedale, Trusha Bhatt, Victoria Corkhill, Victoria de Georgio-Miller, Vijaya Karanam, Yeneit Liew, and Zoey Robinson.

The authors thank the Royal College of Obstetricians and Gynaecologist for supporting this project.

\section{Disclosure}

The authors report no conflicts of interest in this work.

\section{References}

1. Knight M, Kenyon S, Brocklehurst P, Neilson J, Shakespeare J, Kurinczuk JJE. Saving Lives, Improving Mothers' Care Lessons Learned to Inform Future Maternity Care from the UK and Ireland Confidential Enquiries into Maternal Deaths and Morbidity 2009-2012. Oxford, UK: University of Oxford; 2014.

2. Wilkinson H. Saving mothers' lives. Reviewing maternal deaths to make motherhood safer: 2006-2008. BJOG. 2011;118(11): $1402-1403$.

3. Zwart JJ, Dupuis JRO, Richters A, Öry F, van Roosmalen J. Obstetric intensive care unit admission: a 2-year nationwide population-based cohort study. Intensive Care Med. 2010;36(2):256-263.

4. Health and Social Care Information Centre. Hospital Episode Statistics NHS Maternity Statistics - England, 2013-2014; 2015. Available from: http://www.hscic.gov.uk/catalogue/PUB16725/nhs-mate-eng-2013-14summ-repo-rep.pdf. Accessed November 28, 2016.

5. Westhoff G, Cotter AM, Tolosa JE. Prophylactic oxytocin for the third stage of labor to prevent postpartum hemorrhage. Cochrane Database Syst Rev. 2013;10:CD001808 
6. Prendiville WJ, Elbourne D, McDonald S. Active versus expectant management in the third stage of labor (Review). Cochrane Database Syst Rev. 2000;3:CD000007.

7. Royal College of Obstetricians and Gynecologists; Green-top Guideline 52: Prevention and Management of Postpartum Hemorrhage; 2009. Available from: https:/www.rcog.org.uk/globalassets/documents/guidelines/gt52postpartumhaemorrhage0411.pdf. Accessed November 28, 2016.

8. Redshaw M, Rowe R, Schroeder L, et al. Mapping maternity care: the configuration of maternity care in England Birthplace in England research programme. Final report part 3. NIHR Serv Deliv Organ Program. 2011:40.

9. The National Institute for Health and Care Excellence. Intrapartum care for healthy women and babies; 2014. Available from: https://www.nice. org.uk/guidance/cg190?unlid=105541892016715182537. Accessed November 28, 2016.

10. McDonald S, Abbott JM, Higgins SP. Prophylactic ergometrine-oxytocin versus oxytocin for the third stage of labour (Review). Cochrane Database Syst Rev. 2007.
11. Gizzo S, Patrelli TS, Di Gangi S, et al. Which uterotonic is better to prevent the postpartum hemorrhage? Latest news in terms of clinical efficacy, side effects, and contraindications a systematic review. Reprod Sci. 2013;20(9):1011-1019.

12. Gallos ID, Williams HM, Price MJ, et al. Uterotonic agents for preventing postpartum haemorrhage: a network meta-analysis. The Cochrane Library. 2015.

13. Mousa HA, Alfirevic Z. Major postpartum hemorrhage: survey of maternity units in the United Kingdom. Acta Obstet Gynecol Scand. 2002;81(8):727-730.

14. Kramer MS, Berg C, Abenhaim H, Dahhou M, Rouleau J, Mehrabadi A, Joseph KS. Incidence, risk factors, and temporal trends in severe postpartum hemorrhage. Am J Obstet Gynecol. 2013;209(5):449, e1-e7.

15. Dunn TS, Heinrichs GA, Lynch AM. Predicting postpartum hemorrhage. Obstet Gynecol. 2006;107(4):28S.

16. Siassakos D, Fox R, Crofts JF, Hunt LP, Winter C, Draycott TJ. The management of a simulated emergency: better teamwork, better performance. Resuscitation. 2011;82(2):203-206.
Risk Management and Healthcare Policy

\section{Publish your work in this journal}

Risk Management and Healthcare Policy is an international, peer-reviewed open access journal focusing on all aspects of public health, policy, and preventative measures to promote good health and improve morbidity and mortality in the population. The journal welcomes submitted papers covering original research, basic science, clinical and epidemiological
Dovepress

studies, reviews and evaluations, guidelines, expert opinion and commentary, case reports and extended reports. The manuscript management system is completely online and includes a very quick and fair peerreview system, which is all easy to use. Visit http://www.dovepress.com testimonials.php to read real quotes from published authors. 